\title{
Unexpected Adverse Event
}

National Cancer Institute

\section{Source}

National Cancer Institute. Unexpected Adverse Event. NCI Thesaurus. Code C41334.

Any adverse event associated with a medical product or procedure that has not been previously observed, whether or not the event was anticipated because of the pharmacologic properties of the study agent or the nature of the medical procedure. This includes events that are more serious than expected or occur more frequently than expected, particularly, any adverse experience, the nature, severity or frequency of which is not consistent with the product label, or with the current investigator brochure for investig ational agent; or with the risk information described in the investigational plan or protocol or consent form. 